\title{
Super and hyperdeformed states, and reactions to populate them
}

\author{
J. Cseh ${ }^{1, a}$, J. Darai ${ }^{2}$, A. Algora ${ }^{3}$, N. Antonenko ${ }^{4}$, and G. Adamian ${ }^{4}$ \\ 1 Institute of Nuclear Research, Hungarian Academy of Sciences, Debrecen, Pf. 51, Hungary-4001 \\ 2 Institute of Experimental Physics, University of Debrecen, Debrecen, Pf. 105, Hungary-4010 \\ 3 IFIC, CSIC-Universidad de Valencia, A. C. 22085, E 46071, Valencia, Spain \\ 4 Bogoliubov Laboratory, Joint Institute for Nuclear Research, 141980 Dubna, Russia
}

\begin{abstract}
We study the possible binary cluster conf gurations of the superdeformed and hyperdeformed states of some $N=Z$ nuclei. These investigations contribute to the structural understanding of the shape isomers, and indicate some reaction channels to populate them.
\end{abstract}

\section{Introduction}

The investigation of clusterization of a specif c nuclear state is important from two aspects. First it contributes to our understanding of the nuclear structure. Second it gives valuable information on the nuclear reactions, which can populate the state in question. In particular, a binary cluster conf guration with the clusters in their ground intrisic state is uniquely related to a reaction channel, like target and projectile in their ground state. Actually, one of the possible def nition of a binary cluster conf guration is provided by this kind of reaction channel. (Since it is directly related to its observation, probably this is the best way to def ne a cluster conf guration.) Therefore, by studying binary cluster conf gurations of a nuclear state we get a straightforward indication on some possible reaction channels which can reach it.

The investigation of the extremely deformed states, i.e superdeformed (SD), or hyperdeformed (HD) states in nuclei is a long-standing topic. This kind of shape isomers of the $N=Z$ nuclei, however, are known only recently. The experimental observation of the SD states in these nuclei started a decade ago or so, while the question of the hyperdeformation is mainly a topic for theoretical predictions. In this contribution we investigate the $\mathrm{SD}$ and $\mathrm{HD}$ states of $N=Z$ nuclei from a theoretical viewpoint, and discuss some aspects of their experimental observation as well.

\section{Methods of investigation}

Both in our determination of the shape isomers, and in the investigation of their possible clusterizations symmetryconsiderations play an important role. In particular, the quasidynamical (or effective) $U(3)$ symmetry is used [1]. It is a generalization of the concept of the real $U(3)$ symmetry, known to be approximatelly valid in light nuclei [2]. The

\footnotetext{
a e-mail: cseh@atomki.hu
}

quasi-dynamical symmetry is more general in the following sense. The Hamiltonian breaks the symmetry in such a way that the $U(3)$ quantum numbers are not valid for its eigenvectors either (contrary to the case of the real $U(3)$ dynamical symmetry). In other words neither the operator is symmetric (i.e. it is not a $U(3)$ scalar), nor its eigenvectors (i.e. they do not transform according to a single irreducible representation) [3]. Yet, the symmetry is present is some sense.

An asymptotic Nilsson-state serves as an intrinsic state for the quasi-dynamical $S U(3)$ representation. Thus the effective quantum numbers are determined by the Nilssonstates in the regime of large deformation [4]. When the deformation is not large enough, then we can expand the Nilsson-states in the asymptotic basis, and calculate the effective quantum numbers based on this expansion [5].

The $S U(3)$ quantum numbers uniquely determine the quadrupole shape of the nucleus [6], thus we obtain the shape isomers from them. In particular, a self-consistency calculation is performed with respect to the quadrupole shape of nucleus. It is based on the application of the quasidynamical $U(3)$ quantum numbers [7], and in those cases when a detailed comparison can be made with the more traditional energy-minimum calculations, the results are very similar $[8,9,7]$.

Once the shape isomers have been found, the next question is how they are related to cluster conf gurations. To f nd their connection we use the Harvey prescription [10] and the $U(3)$ selection rule [9]. They can incorporate the effects of the exclusion principle, only in an approximate way, of course. But it is a well-def ned way, and its validity can be checked by making a comparison with the results of the fully microscopic description, where they are available. In geometrical terms the $U(3)$ selection rule expresses the similarity of the quadrupole-deformation of the cluster conf guration and the shell-model (or collective model) state.

Energetic preference represents a complementary viewpoint for the selection of clusterization. We incorporate 
it in two different ways: i) by applying simple bindingenergy arguments [11], and ii) by performing double-folding calculations, according to the dinuclear system model $[12$, 13].

\section{Case studies}

\section{$3.1{ }^{36} \mathrm{Ar}$}

The SD band of the ${ }^{36} \mathrm{Ar}$ nucleus was reported in [14]. Following the experimental observation a considerable theoretical effort has been concentrated on this band. In [15] e.g. the possible binary clusterizations of this state was studied sytematically. Similar studies have been done also for the ground, and the hyperdeformed band. The latter one had been predicted from alpha-cluster model calculations [16]. One of the interesting conclusions of this work [15], was that the HD state of the ${ }^{36} \mathrm{Ar}$ nucleus could be populated in the ${ }^{24} \mathrm{Mg}+{ }^{12} \mathrm{C}$ and ${ }^{20} \mathrm{Ne}+{ }^{16} \mathrm{O}$ reactions.

A recent analysis of the ${ }^{24} \mathrm{Mg}+{ }^{12} \mathrm{C}$ elastic scattering [17] revealed the fact that the cross section can be described only by supposing resonances on top of the potential scattering. This very careful analysis incorporated phase-shift study, as well as Regge-pole and energy-dependent resonance calculations. The existence of fve resonances have been proved, which have angular momenta $2,4,6$, 7,8 . These states together with the resonances from the ${ }^{20} \mathrm{Ne}+{ }^{16} \mathrm{O}$ reactions seem to establish a rotational band [17], as shown in the upper part of Fig. 1. Its moment of inertia is in a very good agreement with that of the HD shape predicted from alpha-cluster model [16]. The similarity of the (predicted and observed) moments of inertia, and the fact that the resonances were seen in exactly those reactions, which def ne the preferred cluster-conf gurations of the HD shape suggest that the recently observed band in Fig. 1. is a good candidate for the hyperdeformed shape isomer of the ${ }^{36}$ Ar nucleus. For comparison also the ground and superdeformed bands are indicated in Fig. 1.

Since a candidate for the HD state showed up, the exciting question arises if such a shape can be seen in shellmodel calculation as well. In [7]. we have carried out Nilssonmodel+quasi-dynamical $S U(3)$ calculation in order to f nd the answer. This calculation gives a HD state which has exactly the same symmetry as that from the alpha-cluster model. The moment of inertia from these model agrees well with the one indicated by the experiment.

\section{$3.2{ }^{56} \mathrm{Ni}$}

The stable elongated shapes of the ${ }^{56} \mathrm{Ni}$ nucleus, which are relevant for clusterization, from a similar Nilsson-model + quasi-dynamical $U(3)$ symmetry calculation, is shown in Figure 3. In this f gure it is not the minima, rather the horizontal plateaus, which correspond to the stable shapes. (They are insensitive to the small changes of the input parameter. Furthermore, these deformations fulf ll the selfconsistency argument between the input and output deformation parameters to some approximation.)

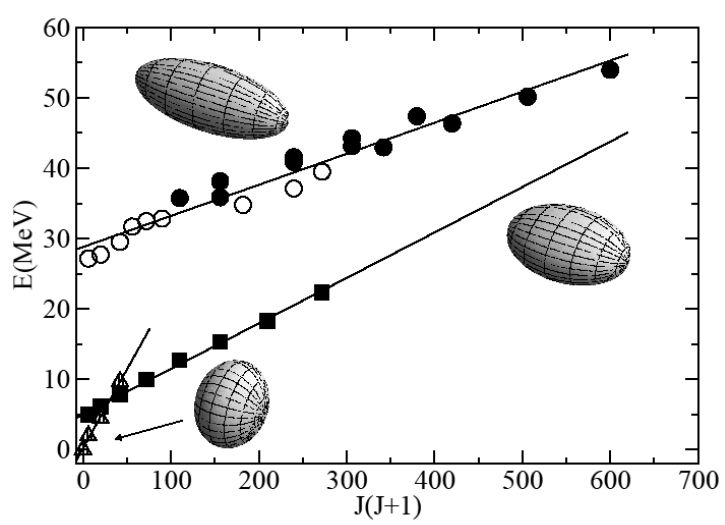

Fig. 1. Excited states in ${ }^{36} \mathrm{Ar}$, obseved as resonances in the ${ }^{12} \mathrm{C}+{ }^{24} \mathrm{Mg}$ (open circles) and ${ }^{16} \mathrm{O}+{ }^{20} \mathrm{Ne}$ (full circles) reactions. The SD band observed in ${ }^{36} \mathrm{Ar}$ and the ground-state band are also included. The shapes are from Nilsson-calculations.

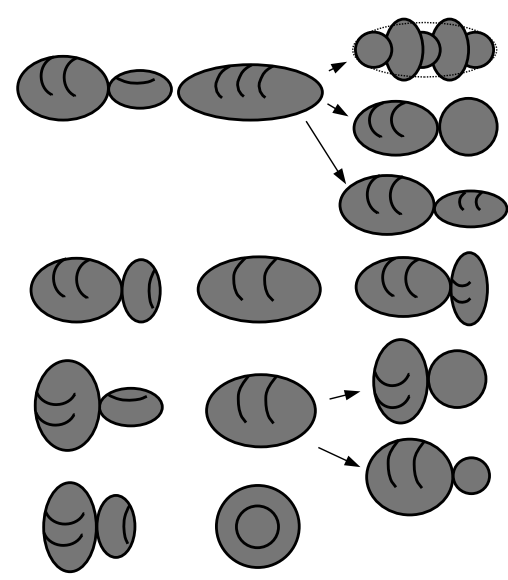

Fig. 2. The shape isomers of the ${ }^{36} \mathrm{Ar}$ nucleus from Nilssonmodel calculations, and their amalgamation from two clusters. The central part shows the shell-model results for the ground (at the bottom), superdeformed, triaxial, and hyperdeformed (at the top) states. The left column corresponds to the ${ }^{24} \mathrm{Mg}+{ }^{12} \mathrm{C}$ clusterization. The right side illustrates the ${ }^{32} \mathrm{~S}+{ }^{4} \mathrm{He},{ }^{20} \mathrm{Ne}+{ }^{16} \mathrm{O}$, ${ }^{28} \mathrm{Si}$ (prolate) $+{ }^{8} \mathrm{Be},{ }^{28} \mathrm{Si}$ (prolate) $+{ }^{8} \mathrm{Be},{ }^{20} \mathrm{Ne}+{ }^{16} \mathrm{O}$ conf gurations (from the bottom), respectively. At the upper most line also the alpha-clusterization of the HD state is shown from the work [16], where the contour corresponds to the HD state of the Nilssonmodel.

As it is seen from the f gure, the triaxial ground-state (for which the experimental deformation is $\beta_{2}=0.173$ ) is followed by a prolate-like deformed state of $0 \hbar \omega$ excitation. The next region of stability corresponds to the superdeformed shape. This state represents 4 nucleon excitation, being very much in line with $[18,19]$. Then appears an even more deformed state with triaxial shape, and two pronounced hyperdeformed shapes close to each other.

It is remarkable that a superdeformed, a triaxial and a hyperdeformed state appear both in the alpha-cluster-model calculation [19], and in our (Nilsson-model-based) quasi-dynamical symmetry consideration. The superdeformed 


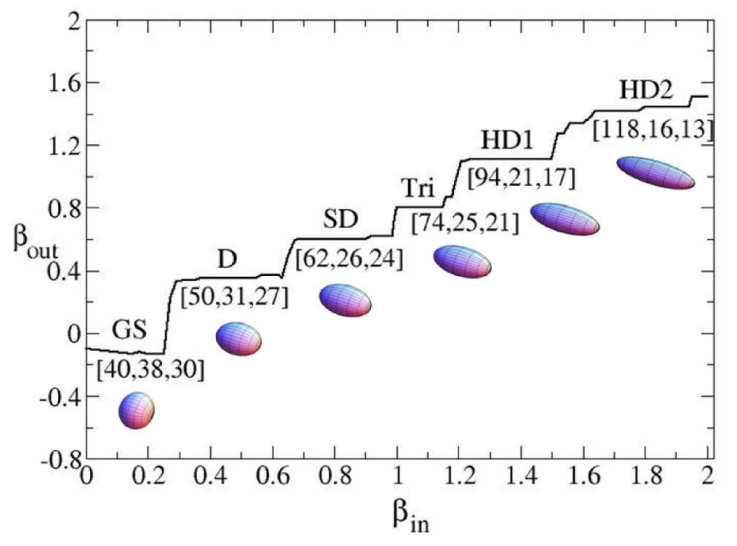

Fig. 3. Quadrupole deformation of the ${ }^{56} \mathrm{Ni}$ nucleus from the Nilsson-model with the effective $U(3)$ quantum numbers and schematic illustrations of the shape at the plateaus.

states seem to correspond to each other exactly, both of them being a $4 \hbar \omega$ excitation. Then we observe a largely deformed triaxial state with $12 \hbar \omega$, which is not completely identical, but similar to that of the alpha-cluster model (with $16 \hbar \omega)$. This latter state is considered to be a candidate for the ${ }^{28} \mathrm{Si}+{ }^{28} \mathrm{Si}$ molecular resonances, in which the two oblate ${ }^{28} \mathrm{Si}$ are thought to have an equator-to-equator position. The alpha-cluster-model gives also a hyperdeformed state, and our calculation have two candidates for that. Based on their possible cluster-structure the lower-lying one seems to be very similar to that of the work [19].

We have found that the ground state of ${ }^{56} \mathrm{Ni}$ prefers asymmetric cluster conf gurations, from among the alphalike clusterization only ${ }^{4} \mathrm{He}+{ }^{52} \mathrm{Fe}$ is allowed. The deformed, superdeformed and largely deformed triaxial states match with several clusterizations. Structure considerations suggest that the correlated ${ }^{28} \mathrm{Si}+{ }^{28} \mathrm{Si}$ and ${ }^{40} \mathrm{Ca}+{ }^{16} \mathrm{O}$ resonances correspond to the superdeformed state of ${ }^{56} \mathrm{Ni}$, but not to the hyperdeformed one. In the latter case the ${ }^{40} \mathrm{Ca}+{ }^{16} \mathrm{O}$ conf guration has a strong structural forbiddenness [20]. The ${ }^{24} \mathrm{Mg}+{ }^{32} \mathrm{~S}$ cluster conf guration on the other hand, which is determined by the entrance channel of the ternary $f$ ssion experiment [21] matches both with the SD and HD states, and with the largely deformed triaxial state in between.

The triaxial state is of special interest, because it is thought to be related to the molecular resonances of two ground-state-like (oblate) ${ }^{28} \mathrm{Si}$ clusters in their equator-toequator conf guration. This conf guration is allowed in the triaxial state from all cited studies. If the equator-to-equator conf guration is not exactly paralell, then other alpha-like binary clusterizations, like e.g. ${ }^{24} \mathrm{Mg}+{ }^{32} \mathrm{~S}$, are also possible.

The hyperdeformed state both from the alpha-cluster and from our Nilsson-calculation prefers a binary conf guration of prolate ${ }^{28} \mathrm{Si}$ clusters with a pole-to-pole conf guration. The state from our quasi-dynamical considerations allows ${ }^{24} \mathrm{Mg}+{ }^{32} \mathrm{~S}$ as well (again close to the position in which the longest major axes of both nuclei are parallel with the molecular axis). The HD state from alpha-cluster studies does not contain this conf guration.

It is an interesting f nding that different states can be built up from the same two clusters, like e.g. two oblate (ground-state-like) ${ }^{28} \mathrm{Si}$ can result in the $0 \hbar \omega$ prolate deformed sates, the superdeformed state with $4 \hbar \omega$ excitation, as well as the largely-deformed triaxial state with many particle-hole excitation. The difference in these cases is the relative orientation of the two deformed clusters. This observation is a consequence of the fact that the Pauliprinciple was taken into account, and no simplifying assumptions were made on the shapes and relative orientations of the clusters.

The energetic preference of the cluster conf gurations were obtained from binding-energy arguments [11], and from calculations based on the Dinuclear System Model [12]. The latter ones were performed both for the poleto-pole conf gurations and for the ones derived from the microscopic considerations. The ${ }^{4} \mathrm{He}+$ core conf guration turned out to be the most preferred one, followed by the ${ }^{8} \mathrm{Be}+$ core one. Then a group of the ${ }^{12} \mathrm{C},{ }^{28} \mathrm{Si}$, and ${ }^{16} \mathrm{O}$ clusters come, with close-lying values, but in different order from different calculations. The ${ }^{24} \mathrm{Mg}$ and ${ }^{20} \mathrm{Ne}$ turned out to be the least-preferred alpha-like clusters.

More detailed investigations on this nucleus is presented in [20]. In this work the results of the energetic calculation with three different methods are compared: in addition to the binding energy, and double folding, mentioned above, there the extended liquid drop model [22] was also applied.

\subsection{Other $N=Z$ nuclei}

We have carried out similar investigations for other $N=Z$ nuclei. For the ${ }^{40} \mathrm{Ca}$ we have discussed the problem of clusterization in [23]. More recently we performed also Nilsson model + quasi-dynamical symmetry calculatiuon for the determination of the shape isomers. Some of the results, together with those concerning the ${ }^{60} \mathrm{Zn}$ nucleus is shown in Table I.

\section{Summary and conclusions}

In this paper we have considered the elongated shape isomers of some $N=Z$ nuclei and their possible binary clusterizations. Both in $\mathrm{f}$ nding the stable shapes and in determining their relations to cluster conf gurations symmetry considerations were applied extensively.

We have determined the shape isomers from the quasidynamical $U(3)$ symmetry, obtained from Nilsson-calculations.

In searching for the possible binary clusterizations of the shape isomers we have taken into account both natural laws which govern the building up of a nucleus from smaller constituents. The exclusion principle was taken into account by applying a selection rule (in combination with Harvey's prescription), based on the microscopic conf guration associated to the quasi-dynamical $U(3)$ symmetry. In this way the Pauli-principle is incorporated only in 
Table 1. Super and hyperdeformed states of some $N=Z$ nuclei. Their shell model excitation quanta, and the possible alpha-like clusterizations (in ground intrinsic state) are given. The $o$, and $p$ subscripts of ${ }^{28} \mathrm{Si}$ refer to oblate and prolate shapes, respectively.

\begin{tabular}{|c|c|c|c|c|}
\hline \multirow[t]{2}{*}{ Nucl. } & \multicolumn{2}{|r|}{ SD } & \multicolumn{2}{|r|}{ HD } \\
\hline & $\hbar \omega$ & $\mathrm{C}_{1}+\mathrm{C}_{2}$ & $\hbar \omega$ & $\mathrm{C}_{1}+\mathrm{C}_{2}$ \\
\hline${ }^{36} \mathrm{Ar}$ & 4 & $\begin{array}{l}{ }^{32} \mathrm{~S}+{ }^{4} \mathrm{He},{ }^{28} \mathrm{Si}_{o, p}+{ }^{8} \mathrm{Be}, \\
{ }^{24} \mathrm{Mg}+{ }^{12} \mathrm{C}\end{array}$ & 12 & $\begin{array}{l}{ }^{28} \mathrm{Si}_{p}+{ }^{8} \mathrm{Be}, \\
{ }^{24} \mathrm{Mg}+{ }^{12} \mathrm{C}, \\
{ }^{20} \mathrm{Ne}+{ }^{16} \mathrm{O}\end{array}$ \\
\hline${ }^{40} \mathrm{Ca}$ & 8 & $\begin{array}{l}{ }^{32} \mathrm{~S}+{ }^{8} \mathrm{Be},{ }^{28} \mathrm{Si}_{o}+{ }^{12} \mathrm{C}, \\
{ }^{20} \mathrm{Ne}+{ }^{20} \mathrm{Ne}\end{array}$ & 16 & $\begin{array}{l}{ }^{28} \mathrm{Si}_{p}+{ }^{12} \mathrm{C}, \\
{ }^{24} \mathrm{Mg}+{ }^{16} \mathrm{O}, \\
{ }^{20} \mathrm{Ne}+{ }^{20} \mathrm{Ne}\end{array}$ \\
\hline${ }^{56} \mathrm{Ni}$ & 4 & $\begin{array}{l}{ }^{48} \mathrm{Cr}+{ }^{8} \mathrm{Be},{ }^{44} \mathrm{Ti}+{ }^{12} \mathrm{C}, \\
{ }^{40} \mathrm{Ca}+{ }^{16} \mathrm{O},{ }^{36} \mathrm{Ar}+{ }^{20} \mathrm{Ne}, \\
{ }^{32} \mathrm{~S}+{ }^{24} \mathrm{Mg},{ }^{28} \mathrm{Si}_{o, p}+{ }^{28} \mathrm{Si}_{o, p}\end{array}$ & 24 & $\begin{array}{l}{ }^{32} \mathrm{~S}+{ }^{24} \mathrm{Mg} \\
{ }^{28} \mathrm{Si}_{p}+{ }^{28} \mathrm{Si}_{p}\end{array}$ \\
\hline${ }^{60} \mathrm{Zn}$ & 4 & $\begin{array}{l}{ }^{52} \mathrm{Fe}+{ }^{8} \mathrm{Be},{ }^{48} \mathrm{Cr}+{ }^{12} \mathrm{C}, \\
{ }^{36} \mathrm{Ar}+{ }^{24} \mathrm{Mg},{ }^{32} \mathrm{~S}+{ }^{28} \mathrm{Si}_{o, p}\end{array}$ & 20 & $\begin{array}{l}{ }^{32} \mathrm{~S}+{ }^{28} \mathrm{Si}_{o, p}, \\
{ }^{36} \mathrm{Ar}+{ }^{24} \mathrm{Mg}\end{array}$ \\
\hline
\end{tabular}

an approximate way, of course. But it is done in a welldef ned procedure, which can be checked in simple systems by comparing with exact results. This approximation can be illustrated in simple geometrical terms, in spite of its abstract algebraic content: it measures, how similar the quadrupole deformations are in the cluster conf guration and in the shell-model (or collective model) state.

The clusters were considered to have a deformation, like the corresponding free nuclei (spherical, prolate, oblate or triaxial), and no constraints were applied for their relative orientation.

The methods we applied here seem to be applicable in heavier nuclei, too. Symmetry considerations can be helpful in studying both the shape isomers of nuclei, and their clusterizations. As for this latter problem is concerned we think that the preferred cluster conf gurations are those ones which are favoured by the energetics, and which are Pauliallowed. From the viewpoint of the application to heavier systems we consider it as a promising sign, that the results of the present method [23] are very similar to the ones from $\mathrm{ab}$ initio calculations for the case of the ${ }^{40} \mathrm{Ca}$ nucleus [24], where the fully microscopic treatment was also applied.

\section{Acknowledgment}

This work was supported by the OTKA (Grant No K72357), TÁMOP (4.2.1./B-09/1/KONV-2010-0007/IK/IT), NKTH (Hungarian-Spanish collaboration project no. ES-26/2008), and MTA-JINR collaboration (project no 2009/001).

\section{References}

1. P. Rochford, D. J. Rowe, Phys. Lett. B 210 (1988) 5; D. J. Rowe, P. Rochford, J. Repka, J. Math. Phys. 29 (1988) 572.

2. J. P. Elliott, Proc. Roy. Soc. A 245 (1958) 128 and 562.
3. J. Cseh, Proc. IV Int. Symp. on Quantum Theory and Symmetries (Varna) (Sof a: Heron Press) (2006) p. 918.

4. M. Jarrio, J. L. Wood, and D. J. Rowe, Nucl. Phys. A 528 (1991) 409.

5. P. O. Hess, A. Algora, M. Hunyadi, J. Cseh, Eur. Phys. J. A 15 (2002) 449.

6. D. J. Rowe, Rep. Prog. Phys. 48 (1985) 1419.

7. J. Cseh et al, Phys. Rev. C 80 (2009) 034320.

8. J. Cseh, J. Darai, A. Algora, H. Yepez-Martinez, P. O. Hess, Rev. Mex. Fis. S. 54 (3) (2008) 30.

9. J. Cseh, J. Darai, AIP Conf. Proc. 1098: Fusion08 225 (2008), and references therein.

10. M.Harvey, Proc. 2nd Int. Conf. on Clustering Phenomena in Nuclei, (College Park) USDERA report ORO4856-26 (1975) p. 549.

11. B. Buck, A. C. Merchant, S. M. Perez, Few-Body Systems 29 (2000) 53.

12. V.V. Volkov, Phys. Rep. 44 (1978) 93; Deep inelastic nuclei reactions (Energoizdat, Moscow, 1982).

13. T. M. Schneidman et al., Phys. Lett. B 526 (2002) 322.

14. C.E. Svensson et al, Phys. Rev. Lett. 85 (2000) 2693.

15. J. Cseh, A. Algora, J. Darai, P.O. Hess, Phys. Rev. C70 (2004) 034311.

16. W.D.M. Rae, A.C. Merchant, Phys. Lett. B279 (1992) 207.

17. W. Sciani et al, Phys. Rev. C80 (2009) 034319.

18. D. Rudolph et al., Phys. Rev. Lett. 823763 (1999).

19. J. Zhang, A. C. Merchant, W. D. M. Rae, Phys. Rev. C49 562 (1994).

20. J. Darai et al., to be published.

21. W. von Oertzen et al., Eur. Phys. Jour. A36 (2008) 279.

22. G. Royer, B. Remaud, Nucl. Phys. A 444 (1985) 477.

23. J. Cseh et al., Rev. Mex. Fis. S. 52 (4) (2006) 11.

24. Y. Kanada-Enyo, M. Kimura, H. Horiuchi, AIP Conf. Proc. 644 (2003) 188. 